\title{
Aspects of a dynamical gluon mass approach to elastic hadron scattering at LHC
}

\author{
D. A. Fagundes ${ }^{\text {a,* }}$, E. G. S. Luna ${ }^{\text {b }}$, M. J. Menon ${ }^{\text {a }}$, A. A. Natale ${ }^{c}$ \\ ${ }^{a}$ Instituto de Física Gleb Wataghin, Universidade Estadual de Campinas, UNICAMP, CEP 13083-859, Campinas, SP, \\ Brazil \\ ${ }^{b}$ Instituto de Física, Universidade Federal do Rio Grande do Sul, Caixa Postal 15051, CEP 91501-970, Porto Alegre, RS, \\ Brazil \\ ${ }^{c}$ Instituto de Física Teórica, UNESP - Universidade Estadual Paulista, Rua Dr. Bento T. Ferraz, 271, Bloco II 01140-070,
} São Paulo - SP, Brazil

\begin{abstract}
We discuss how the main features of the recent LHC data on elastic scattering can be described by a QCD-inspired formalism with a dynamical infrared mass scale. For this purpose new developments on a dynamical gluon mass approach are reported, with emphasis on a method to estimate uncertainty bounds in the predictions for the high-energy scattering observables. We investigate the effects due to the correlations among the fixed and free parameters involved and show that the band of predictions are consistent with the recent data from the TOTEM experiment, including the forward quantities and the differential cross section up to the dip position.
\end{abstract}

Keywords: Elastic scattering, Total cross sections, Hadron-induced high- and super-high-energy interactions.

\section{Contents}

1 Introduction

2 Formalism

2.1 Physical Quantities and Eikonal Representation . . . . . . . . . . . . . . . . 2

2.2 Dynamical Gluon Mass and Gluon-Gluon Cross Section _ . . . . . . . . . . . . . . 3

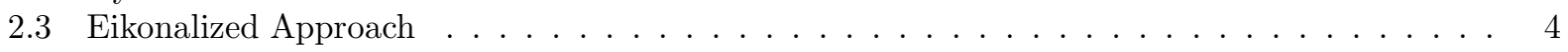

3 Testing parameters: Fits and Results 6

3.1 Data Ensemble and Fit Procedure . . . . . . . . . . . . . . . . . . . . 7

3.2 Testing Parameters . . . . . . . . . . . . . . . . . . . . . . . . .

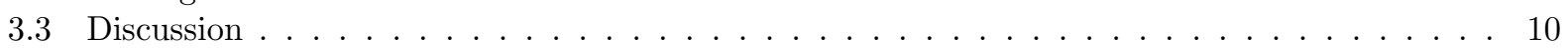

4 Conclusions and Final remarks

\section{Introduction}

The TOTEM experiment has been designed to study elastic and diffractive scattering at the LHC, providing information on the $p p$ total cross sections and the elastic differential cross sections at the energy

\footnotetext{
${ }^{*}$ Corresponding author

Email addresses: fagundes@ifi.unicamp.br (D. A. Fagundes), luna@if.ufrgs.br (E. G. S. Luna), menon@ifi.unicamp.br (M. J. Menon), natale@ift.unesp.br (A. A. Natale) 
region $7-14 \mathrm{TeV}$ and therefore expected to represent a crucial source of information for selecting models and pictures. First results at $7 \mathrm{TeV}$ have already been published showing that no model predictions present full agreement with both total cross section and the differential cross section up to $2.5 \mathrm{GeV}^{2}$ [1, 2] .

As a consequence reviewing processes are in order in the phenomenological context, and among the variety of approaches [3, 4] , the QCD-inspired models certainly play a fundamental role. However, although this class of models presents explicit connections with minijets and/or semihard concepts, where some kind of perturbative technique may be applied, the intrinsic soft character involved in the elastic scattering demands a quantitative connection with the non-perturbative QCD.

With this aim (see also [5, 6, , 7]), a novel interpretation for some fundamental parameters in eikonalized QCD-inspired models has been previously proposed, allowing a quantitative connection between elastic hadron scattering data and the nonperturbative regime [8, 9, 10]. This Dynamical Gluon Mass (DGM) approach is based on the possibility that the nonperturbative dynamics of QCD generates an effective gluon mass at the small momentum transfer region, which is strongly supported by recent QCD lattice simulations [11], as well as by other phenomenological results [12, 13, 14]. In this context, the increase of the hadronic total cross sections is managed by the interplay between the gluon distribution function and a gluon mass scale and previous analysis on $p p$ and $\bar{p} p$ elastic scattering has indicated good descriptions of the forward data up to $\sqrt{s}=1.8 \mathrm{TeV}$, including the differential cross section at small momentum transfer 9 .

However, as commented above, the new results by the TOTEM collaboration demands further tests and developments in the phenomenological context and that is the point we are interested in here. As we shall show and discuss, even not using these new results as input information for quantitative fit procedures, general aspects of our formalism can be revisited and improved in the same energy region investigated before, namely between $10 \mathrm{GeV}$ and $1.8 \mathrm{TeV}$ (some preliminary ideas on these respect have already been discussed in 15]). Specifically, in this work we present new developments related to some formal and practical aspects of the approach with focus on: (1) quantitative account of the energy dependencies in the coupling constant and dynamical gluon mass; (2) fits based on improved data ensemble; (3) detailed analysis on the influence in the evaluated quantities associated with two fundamental parameters, the dynamical gluon mass scale and the soft Pomeron intercept; (4) estimation of bounds in all evaluated quantities associated with relevant physical intervals for the above parameters; (5) explicit reference to some calculational details. We shall show that these parameters play a crucial role in the energy region above that used in the fit procedures, which means they can not be fixed at ad hoc values without a fundamental physical justification. By considering relevant numerical intervals for the dynamical gluon mass scale and the soft Pomeron intercept, the question associated with the evaluation of the corresponding (statistical) uncertainty regions is addressed and a solution based on bounds for the high-energy predictions is proposed. Within these uncertainty bands we show that the recent data obtained by the TOTEM Collaboration at $\sqrt{s}=7 \mathrm{TeV}$ are reasonably well described, except the differential cross section beyond the dip position. Predictions at $\sqrt{s}=14 \mathrm{TeV}$ and cosmic-ray energy region (Auger) are also presented. The advantages and some drawbacks associated with this formalism are discussed and further necessary developments outlined as well.

The text is organized as follows. In Sect. 2 we treat the theoretical context, with explicit reference to novel formal account of the QCD scale and some calculational aspects. In Sect. 3 the analysis on the influence of the dynamical gluon mass and the soft Pomeron intercept are presented in detail, as well as the fit procedures, the proposed method for uncertainty inferences, the predictions at $7 \mathrm{TeV}, 14 \mathrm{TeV}$ (LHC), $57 \mathrm{TeV}$ (Auger) and a discussion on all the obtained results. The conclusions and some critical remarks are the contents of Sect. 4.

\section{Formalism}

\subsection{Physical Quantities and Eikonal Representation}

Our analysis is based on three physical quantities expressed in terms of the elastic scattering amplitude $A(s, q)$, where $q=\sqrt{-t}$, with $s$ and $t$ the Mandesltam variables: the differential cross section

$$
\frac{d \sigma}{d q^{2}}(s, q)=\pi|A(s, q)|^{2},
$$


the total cross section

$$
\sigma_{\text {tot }}(s)=4 \pi \operatorname{Im} A(s, q=0)
$$

and the $\rho$ parameter,

$$
\rho(s)=\frac{\operatorname{Re} A(s, q=0)}{\operatorname{Im} A(s, q=0)} .
$$

From (1) and (2) the integrated elastic and inelastic cross sections can be obtained,

$$
\sigma_{\mathrm{el}}(s)=\int_{0}^{\infty} \frac{d \sigma}{d q^{2}}(s, q) d q^{2}, \quad \sigma_{\mathrm{in}}(s)=\sigma_{\mathrm{tot}}(s)-\sigma_{\mathrm{el}}(s) .
$$
[16]

In the eikonal representation (azimuthal symmetry assumed) the scattering amplitude is expressed by

$$
A(s, q)=\mathrm{i} \int_{0}^{\infty} b d b J_{0}(q b)\left\{1-e^{\mathrm{i} \chi(s, b)}\right\}
$$

where $b$ is the impact parameter, $J_{0}$ the zero-order Bessel function and $\chi(s, b)$ the complex eikonal function in the impact parameter space. Eikonal models can be classified or distinguished according to different choices for the eikonal either in the impact parameter space or in the momentum transfer space,

$$
\tilde{\chi}(s, q)=\int_{0}^{\infty} b d b J_{0}(q b) \chi(s, b)
$$

where it is connected with the specific dynamical assumptions that characterize each model. Under the physical condition $\operatorname{Im} \chi(s, b) \geq 0$ this representation constitutes an automatic unitarized framework suitable for theoretical developments. In special, in the case of QCD based or inspired models the eikonal in the momentum transfer space is expressed in terms of hadronic form factors and elementary cross sections and the main point is how to connect these quantities with parton-parton scattering processes. Since in these models the rise of the total cross section at high energies is driven by gluon-gluon semihard scattering, this is the main process to be investigated.

\subsection{Dynamical Gluon Mass and Gluon-Gluon Cross Section}

In addition to lattice QCD [11], the Schwinger-Dyson equations constitutes a fundamental nonperturbative formalism in the investigation of hadron physics, specially in what concerns confinement and dynamical chiral symmetry breaking. Infrared finite solutions for these equations have been obtained by Cornwall by means of the pinch technique leading to a gluon propagator which carries a dynamical mass [17, 18, 19]. A review on this dynamical gluon mass generation is presented in [20], discussions and more details can be found in [9] and references therein. Here we display the main formulas connected with the nonperturbative gluon-gluon cross section, stressing a formal improvement regarding the previous work [9].

In the DGM approach the nonperturbative integrated elastic cross section for the scattering $g g \rightarrow g g$, expressed in terms of the subprocess energy $\hat{s}$, is given by [9]

$$
\hat{\sigma}_{g g}(\hat{s})=\left(\frac{3 \pi \bar{\alpha}_{s}^{2}}{\hat{s}}\right)\left[\frac{12 \hat{s}^{4}-55 M_{g}^{2} \hat{s}^{3}+12 M_{g}^{4} \hat{s}^{2}+66 M_{g}^{6} \hat{s}-8 M_{g}^{8}}{4 M_{g}^{2} \hat{s}\left[\hat{s}-M_{g}^{2}\right]^{2}}-3 \ln \left(\frac{\hat{s}-3 M_{g}^{2}}{M_{g}^{2}}\right)\right]
$$

where $\bar{\alpha}_{s}=\bar{\alpha}_{s}(\hat{s})$ and $M_{g}=M_{g}(\hat{s})$ are the running coupling constant and the dynamical gluon mass, respectively. From the Cornwall solution for the gluon propagator in the case of pure gauge QCD, these quantities are expressed by [17, 18, 19]

$$
\bar{\alpha}_{s}(\hat{s})=\frac{4 \pi}{\beta_{0} \ln \left[\left(\hat{s}+4 M_{g}^{2}(\hat{s})\right) / \Lambda^{2}\right]},
$$




$$
M_{g}^{2}(\hat{s})=m_{g}^{2}\left[\frac{\ln \left(\frac{\hat{s}+4 m_{g}^{2}}{\Lambda^{2}}\right)}{\ln \left(\frac{4 m_{g}^{2}}{\Lambda^{2}}\right)}\right]^{-12 / 11},
$$

where $\beta_{0}=11-\frac{2}{3} n_{f}\left(n_{f}\right.$ is the number of flavors), $\Lambda=\Lambda_{Q C D}$ and $m_{g} \equiv M_{g}(0)$ is the gluon mass scale. A fundamental consequence of this result is its extension to perturbative QCD. In fact, in the limit $\hat{s} \gg \Lambda^{2}$ we have $M_{g}^{2}(\hat{s}) \rightarrow 0$ and $\bar{\alpha}_{s}$ matches the one-loop perturbative QCD one [9].

In this context, by considering $n_{f}=4$ and $\Lambda=284 \mathrm{MeV}$, the only unknown quantity in the evaluation of $\hat{\sigma}_{g g}(\hat{s})$ is the gluon mass scale $m_{g}$, the IR value for $M_{g}^{2}(\hat{s})$. Phenomenological analyses suggest typical values of $m_{g}$ in the range from $300 \mathrm{MeV}$ up to $700 \mathrm{MeV}$ [17, 18, 19, 21, 22, 12, 13, 14] and in [9] it has been inferred $m_{g}=400 \mathrm{MeV}$. To investigate the relevant numerical interval for $m_{g}$ and the effects in the analysis of elastic hadron scattering is one of the main points we are interested in here.

It should be noted that in previous works $[8,9,10,13]$ the dependence of $M_{g}^{2}$ on the energy of the subprocess $g g \rightarrow g g$ has been neglected as a first approximation, that is, in Eq. (6) the IR value $m_{g}^{2}$ has been used in place of $M_{g}^{2}(\hat{s})$. Therefore, the integrated cross section as given by Eq. (6) generalizes the one considered in [8, 9, 13], enlarging the physical meaning of the dynamical gluon mass and the efficiency of the formalism in the data analyses, as shown in Sect. 3. Another parametrization for $M_{g}^{2}(\hat{s})$ is discussed in Appendix A.

\subsection{Eikonalized Approach}

At high energies the soft and the semihard components of the scattering amplitude are closely related [23, 24], and it becomes important to distinguish between semihard gluons, which participate in hard partonparton scattering, and soft gluons, emitted in any given parton-parton QCD radiation process. Hence a formalism based on QCD has to incorporate soft and semihard processes in the treatment of high-energy hadronic interactions and, more importantly, has to bring up information about the infrared properties of QCD.

In the phenomenological context, the developments of the mini-jet or semi-hard QCD picture [3] constitute an interesting step in the search for connections between hadron scattering and QCD subprocesses, associated with gluon-gluon $(g g)$, quark-gluon $(q g)$ and quark-quark $(q q)$ interactions. That is the case in the formalisms by Margolis et al. [25, 26, 27] and the more recent version sometimes referred to as Aspen model, by Block et al. [28, 29]. However, despite its efficiency in the description of the forward quantities in the elastic channel, as well as differential cross sections at small values of the momentum transfer a serious drawback with the Aspen model concerns the absence of the expected connections with nonperturbative QCD. Moreover, and as a consequence, two fundamental quantities, the infrared mass scale, denoted $m_{0}$, and the coupling constant $\alpha_{s}$, are unknown parameters fixed to ad hoc values $600 \mathrm{MeV}$ and 0.5 respectively, in order to obtain best fits in data analyses [28, 29].

In this respect, the main ingredient in the DGM approach [8, 9] has been the novel physically motivated interpretation for both quantities through the gluon mass scale as a natural regulator of the infrared divergences associated with the semihard gluon-gluon subprocess cross sections, leading therefore to direct connections with nonperturbative QCD. The general formulation is largely based on the eikonal structure of the Aspen model [28, 29], but with the above well founded connection, as well as differences regarding free/fixed parameters, constraints and specific parametrization. The point is to consider Eqs. (6 - 8) for the $g g$ subprocess and the IR values $\bar{\alpha}_{s}(\hat{s}=0)$ and $M_{g}^{2}(\hat{s}=0)=m_{g}^{2}$ in substitution to $\alpha_{s}$ and $m_{0}$ in all elementary subprocesses.

Specifically, for $p p$ and $\bar{p} p$ elastic scattering, the complex eikonal in the impact parameter space is expressed in terms of even $(e)$ and odd $(o)$ functions,

$$
\chi_{\bar{p} p}(s, b)=\chi_{e}(s, b)+\chi_{o}(s, b), \quad \chi_{p p}(s, b)=\chi_{e}(s, b)-\chi_{o}(s, b) .
$$

The odd part is expected to account for the difference between both reactions at low energies and to vanish in the high-energy limit. It is therefore parametrized as a Reggeon contribution and in a factorized 
form, with the inclusion of the dynamical gluon mass ingredients and the odd prescription,

$$
\chi_{o}(s, b)=k C_{o} \frac{m_{g}}{\sqrt{s}} \mathrm{e}^{i \pi / 4} W\left(b ; \mu_{o}\right),
$$

where

$$
k \equiv \frac{9 \pi \bar{\alpha}_{s}^{2}(0)}{m_{g}^{2}},
$$

$W\left(b ; \mu_{o}\right)$ is the Fourier transform of the dipole form factor

$$
W\left(b ; \mu_{o}\right)=\frac{\mu_{o}^{2}}{96 \pi}\left[\mu_{o} b\right]^{3} K_{3}\left(\mu_{o} b\right)
$$

( $K_{3}$ is the modified Bessel function of second kind), and $\mu_{o}$ and $C_{o}$ are free fit parameters.

The even part is supposed to be expressed in terms of contributions from elementary interactions, namely gluon-gluon, quark-gluon and quark-quark, in a factorized form at the constituent level,

$$
\begin{aligned}
\chi_{e}(b, s) & =\chi_{q q}(b, s)+\chi_{q g}(b, s)+\chi_{g g}(b, s) \\
& =\mathrm{i}\left[\sigma_{q q}(s) W\left(b ; \mu_{q q}\right)+\sigma_{q g}(s) W\left(b ; \mu_{q g}\right)+\sigma_{g g}(s) W\left(b ; \mu_{g g}\right)\right],
\end{aligned}
$$

where, for $i j=q q, q g, g g, W\left(b, \mu_{i j}\right)$ are overlap functions with the same structure of Eq. $(12), \mu_{i j}$ are free fit parameters and $\sigma_{i j}$ are the corresponding elementary cross sections.

For the sub-processes involving quarks, the parametrizations for the cross sections follows from approximate forms of distribution functions involving quarks and gluons at small $x$ region, with the even prescription in the corresponding eikonal:

$$
\begin{gathered}
\sigma_{q q}(s)=k C_{q q} \frac{m_{g}}{\sqrt{s}} \mathrm{e}^{i \pi / 4}, \\
\sigma_{q g}(s)=k\left\{C_{q g}+C_{q g}^{\prime}\left[\ln \left(\frac{s}{m_{g}^{2}}\right)-i \frac{\pi}{2}\right]\right\},
\end{gathered}
$$

where $C_{q q}, C_{q g}, C_{q g}^{\prime}$, are free fit parameters. The convenience of the general structure adopted in the parametrizations (10), (13), (14) and (15) can be traced back to the early work of Margolis et al. [25, 26, 27] and references therein.

The full $g g$ cross section is expressed by

$$
\sigma_{g g}(s)=C_{g g} \int_{4 m_{g}^{2} / s}^{1} d \tau F_{g g}(\tau) \hat{\sigma}_{g g}(\hat{s}),
$$

where $\tau=\hat{s} / s, F_{g g}(\tau)$ is the convoluted gluon distribution function,

$$
F_{g g}(\tau)=[g \otimes g](\tau)=\int_{\tau}^{1} \frac{d x}{x} g(x) g\left(\frac{\tau}{x}\right),
$$

and $\hat{\sigma}_{g g}(\hat{s})$ represents the subprocess $g g \rightarrow g g$ cross section, given here by the nonperturbative result (6). For the gluon distribution function, since in the DGM approach the small- $x$ semihard gluons play a central role, the phenomenological parametrization used in [28] is also considered here,

$$
g(x)=N_{g} \frac{(1-x)^{5}}{x^{J}}
$$

which simulates the effect of scaling violations in the small $x$. Here $J$ can be associated with the soft Pomeron intercept $(J=1+\epsilon)$. Notice that the ansatz (18) satisfies the normalization condition $\int_{0}^{1} d x x g(x)=A$, 
where $A$ is a constant [34]. If $A=1 / 2, N_{g}=(6-\epsilon)(5-\epsilon) \ldots(1-\epsilon) / 240$. The power 5 appearing in the term $(1-x)$ is suggested by dimensional counting rules [34, 35, 36].

The integration of Eq. (16), taking account of the analyticity properties, demands some comments. The odd contribution to the eikonal, Eq. (10) and those from $q g$ and $q q$ subprocesses to the even eikonal, Eqs. (14) and (15), are closed analytical forms, so that the corresponding prescriptions are straightforward to be applied, leading to the generation of the real and imaginary parts. On the other hand, the $g g$ contribution, Eq. (16), cannot be analytically evaluated and any numerical approach, embodying analyticity, demands some assumptions. We have used two independent methods. In the first one generated numerical points for the real primitive of the integrand in (16) have been parametrized by polynomials in $\ln s$ and then the even prescription has been applied. In the second one we have used first order derivative dispersion relation for even functions [37] applied as

$$
\operatorname{Re} \sigma_{g g}(s) \approx \frac{\pi}{2} \frac{d}{d \ln s} \operatorname{Im} \sigma_{g g}(s),
$$

and numerical derivation of the generated points. $\operatorname{In}(19), \operatorname{Im} \sigma_{g g}(s)$ corresponds to the generated numerical points for the real primitive of (16). Both methods lead to the same results, except for negligible differences at low energies.

With all the above ingredients the formalism is complete. Specifically, it has two fundamental parameters, the dynamical gluon mass scale $m_{g}$ and the soft Pomeron intercept $\epsilon$. Once $m_{g}$ and $\epsilon$ are chosen, the model has 9 free parameters to be determined by fits to the experimental data: 5 normalization constants associated with the odd contribution and elementary cross section $\left(C_{o}, C_{q q}, C_{q g}, C_{q g}^{\prime}, C_{g g}\right)$ and 4 coming from the corresponding form factors $\left(\mu_{o}, \mu_{q q}, \mu_{q g}, \mu_{g g}\right)$. That should be contrasted with 6 fixed and 6 fit parameters in the Aspen model [28, 29].

In what concerns the momentum transfer space, the essential ingredient in the structures of the eikonals (9), (10) and (13) is the combination of four dipole form factors, of type (12) in the impact parameter space (in fact, a linear combination at each fixed energy). Therefore, the dependence of the physical quantities on the momentum transfer is a direct consequence of these choices and in this respect the following comments are in order. Historically, the dipole ansatz may be traced back to the conjecture by Wu and Yang that the distribution of hadronic matter inside a hadron is similar in electromagnetic and strong interactions [30]. Specifically they conjectured that the elastic $p p$ differential cross section might be proportional to the fourth power of the proton charge form factor, that is the form factor measured in electron-proton scattering. In the context of the Chou-Yang model [31], with an input for the form factor, the differential cross section can be evaluated. The use of a parametrization as a sum of exponential in $q^{2}$ by Chou and Yang [32] and the dipole electric form factor by Durand and Lipes [33],

$$
G_{D}(q)=\frac{1}{\left[1+q^{2} / \mu^{2}\right]^{2}}, \quad \mu^{2}=0.71 \mathrm{GeV}^{2},
$$

led to the prediction of the diffractive pattern in $p p$ differential cross section and, more importantly, the correct position of the dip, as experimentally observed latter. However, the dipole parametrization predicts multiple dips and peaks that have never been observed in the experimental data. In our case (as well as in the Aspen model), the oscillatory behaviour, characteristic of the dipole structure can be attenuated by the combination of four different dipoles, at least up to the intermediate region of the momentum transfer (dip-bump structure). However, beyond this region, changes of slopes are expected, as indicated by the results presented in the following section. This effect seems not to be present in the published plots by the TOTEM Collaboration [1], suggesting that other form factors should be regarded in order to describe the high- $t$ data. We shall return to this point in the conclusions and final remarks.

\section{Testing parameters: Fits and Results}

Despite the suitable formal connection with nonperturbative QCD and the efficiency in the description of the experimental data, one important aspect to be investigated in the DGM approach concerns the influence 
of the fundamental parameters in the evaluated quantities (fits and predictions): $m_{g}$ and $\epsilon$. They could certainly be fixed, as done for example with the corresponding parameters (and others) in the Aspen model [28]. However, as we shall show, relevant numerical intervals for $m_{g}$ and $\epsilon$ affect the model predictions at the highest energies and that puts serious limits on the reliability of models with ad hoc values for the fixed parameters involved. Our goal here is to point out the relevance of this aspect and the fact that it demands some solution or a clear explanation, prior to consider these models as physically well founded. To this end we first discuss some aspects of the data ensemble used here (which differs from the one considered in [8, 9]) and outline the fit procedure (Subsection 3.1). Then we treat in some detail the selection of the relevant intervals for the parameters and the proposed methodology for uncertainty inferences (Subsection 3.2), followed by a critical discussion on the obtained results (Subsection 3.3).

\subsection{Data Ensemble and Fit Procedure}

In the previous work [9] the data ensemble used in the fits includes only forward quantities, $\sigma_{\text {tot }}, \rho$ parameter and the slope parameter $B$ up to $1.8 \mathrm{TeV}$. Here we shall consider a different ensemble for the reasons that follows.

Even in the very forward direction the evaluation of the slope parameter depends somehow on the momentum transfer interval considered (at least two points), which renders difficult comparison among results from different experiments with different intervals. That is clearly illustrated in the comparison of the recent result by the TOTEM Collaboration with the existing data 38] that includes the pp2pp result at $200 \mathrm{GeV}$ [39]. Moreover, in a model context the slope is evaluated at one point, $q^{2}=0$, and therefore some bias may always be present in the calculation. For that reason, we shall not consider here the forward slope as input quantity in the data reductions.

On the other hand, as constructed, the formalism is expected to be applicable in the soft region, which in terms of the differential cross section means intervals in momentum transfer roughly up to $1 \mathrm{GeV}^{2}$, or even beyond. The presence of four dipole form factors in the eikonal (Subsection 2.3) corroborates this expectation. Hence we include in the ensemble to be fitted the differential cross section data available at the highest energies, namely $\bar{p} p$ scattering at $546 \mathrm{GeV}$ and $1.8 \mathrm{TeV}$, covering the region up to $q^{2} \sim 1.5 \mathrm{GeV}^{2}$ and $\sim 0.6 \mathrm{GeV}^{2}$, respectively.

Since the numerical tables on the differential cross section at $7 \mathrm{TeV}$ are not yet available and a second measurement of the total cross section (through a luminosity independent method) is expected, we shall not consider here the recent TOTEM results as input in the fits. However, the numerical information available (and published) will be referred to as a guidance in the discussion of our predictions.

Our ensemble therefore includes the $p p$ and $\bar{p} p$ data on $\sigma_{\text {tot }}$ and $\rho$ above $10 \mathrm{GeV}$ up to $1.8 \mathrm{TeV}$, together with the $\bar{p} p$ differential cross section data at $546 \mathrm{GeV}$ and $1.8 \mathrm{TeV}$. The data have been collected from the PDG [40] and Durham [41] sites and in all cases the statistic and systematic errors have been added in quadrature. The data reductions consist in assuming some input values for $\mathrm{m}_{g}$ and $\epsilon$ and through Eqs. (1) to (3) to fit the above data ensemble. For the fit program we have used the class TMinuit of the CERN ROOT Framework [42] and the MIGRAD minimizer, setting the confidence level at $90 \%$.

\subsection{Testing Parameters}

Preliminary results with the above ensemble and fit procedure have already been presented in our previous analysis [15]. The main conclusion has been that by considering narrow intervals for $m_{g}$ and $\epsilon$ all the fitted data are quite well described in all cases. However, at higher energies the predictions are sensitive to each numerical interval considered. Here, we address the question on how to infer quantitative uncertainties in the model predictions taking into account relevant intervals for $m_{g}$ and $\epsilon$. It is important to stress that our point does not concern standard propagation from the errors in the fit parameters, as given by the error matrix (variances and covariances). Our focus is only on the effects of physically motivated intervals for $m_{g}$ and $\epsilon$.

However that is not an easy task, due to the numerical integrations involved, the absence in this case of a statistical method to extract uncertainties and the strong correlations among $m_{g}, \epsilon$ and all the free fit parameters, as we shall shown. What we propose here concerns a method to infer bounds in the evaluated 
quantities in accordance with relevant intervals for $m_{g}$ and $\epsilon$, in a similar way as has been recently done by Achilli et al. in the context of minijet models [43].

Due to the correlations among $\epsilon, m_{g}$ and the fit parameters, a relevant interval for one of the fundamental parameters depends on the relevant interval for the other one. For that reason we first treat $\epsilon$ and then $m_{g}$. For the soft Pomeron intercept, as in [15], we consider the results from the detailed analyses on the extrema and constrained bounds for the intercept, since they are based on both scattering data [44] and scattering together with spectroscopy data [45], respectively. With these bounds, namely $\epsilon_{\text {lower }}=0.080$ and $\epsilon_{\text {upper }}=$ 0.090 [44, 45], we consider as relevant interval all real values in this range, including the bounds. Thus we use the following notation for intervals with that meaning (equally likely values):

$$
\epsilon:[0.080,0.090]
$$

For the dynamical gluon mass scale, as commented in Subsection 2.2, phenomenological analyses suggest $m_{g}$ values in the range from $300 \mathrm{MeV}$ up to $700 \mathrm{MeV}$. Taking into account the interval for $\epsilon$, as we shall show, the following interval for $m_{g}$, larger than that considered in [15], and with the above notation and meaning, is adequate to our purposes:

$$
m_{g}(\mathrm{MeV}):[300,600] .
$$

Once selected the relevant intervals, the next step is to investigate their effects in the fit results and evaluated quantities, looking for the corresponding uncertainties in these quantities. At this point it is important to stress that, in principle, any numerical pair $\left(\epsilon, m_{g}\right)$ in the selected intervals is equally likely and therefore we can not attribute central values with uncertainty, as for example $0.085 \pm 0.005$ for $\epsilon$ and $450 \pm 150$ for $m_{g}$. That is, there is no meaning in assuming that a most probable value is any closer to the middle of this range that it is to either endpoint. Moreover, it is also not possible to test the effects of all real pairs $\left(\epsilon, m_{g}\right)$. For those reasons we have considered a method consisting of the following steps:

1. From different tests with real pairs $\left(\epsilon, m_{g}\right)$ we have selected relevant values for each parameters in each interval, with the result:

$$
\begin{aligned}
\epsilon & : \quad 0.080,0.085,0.090 \\
m_{g} & : \quad 300,400,500,600 \mathrm{MeV}
\end{aligned}
$$

2. For each of the above pairs $\left(\epsilon, m_{g}\right)$, therefore 12 variants, we have performed the data reductions (Subsection 3.1), determining in each case the $\chi^{2}$ per degrees of freedom (DOF) as a test for goodness of fit.

3. Based on the above statistical information and in the global description of the fitted data, we have determined an optimum value for one of the parameters together with the relevant interval for the other one.

4. By fixing one parameter in its optimum value the extrema values of the interval corresponding to the other parameter have been used as upper and lower bounds in the evaluated quantities.

5. The band limited by the above upper and lower bounds has been considered as an estimation of the uncertainty region in the evaluated quantities.

With this strategy we do not take account of the simultaneous influences of both intervals, but only the effect of the interval in one parameter for a fixed value of the other parameter. We shall return to this point in subsection $\mathrm{C}$.

The values of the $\chi^{2} / \mathrm{DOF}$ for each one of the 12 variants are displayed in Table I and plotted in Fig. 1 , in terms of the dynamical gluon mass scale and for each value of the soft Pomeron intercept. Since we have 318 DOF the best results demand $\chi^{2} /$ DOF closest and/or below 1 . Figure 1 plays a central role in our analysis since we can extract the following main conclusions: 
Table 1: Values of the $\chi^{2} / \mathrm{DOF}$ for 318 DOF obtained in all fits.

\begin{tabular}{c|cccc}
\hline & & $m_{g}$ & $\mathrm{MeV}$ & \\
$\epsilon$ & 300 & 400 & 500 & 600 \\
\hline 0.080 & 1.160 & 0.9487 & 0.9690 & 0.9984 \\
0.085 & 0.9899 & 0.9536 & 0.9536 & 1.432 \\
0.090 & 1.001 & 0.9597 & 1.070 & 4.339 \\
\hline
\end{tabular}

1. For $m_{g}=600 \mathrm{MeV}$ the cases $\epsilon=0.085$ and 0.090 are excluded on statistical grounds.

2. For $\epsilon=0.080$, despite the reasonable result at $m_{g}=300 \mathrm{MeV}$, the whole interval in $m_{g}$ corresponds to quite good statistical results.

3. For $m_{g}=400 \mathrm{MeV}$ the whole interval for $\epsilon$ corresponds to quite good statistical results.

4. For both parameters considered simultaneously the intervals $\epsilon$ : $[0.080,0.090]$ and $m_{g}(\mathrm{MeV})$ : [400, 500] correspond to acceptable statistical results.

Despite the last conclusion, in order to develop here a feasible and economic method for uncertainty inferences, we do not consider both intervals simultaneously. For individual intervals, based on the first three statistical conclusions and after checking the descriptions of the fitted data in all cases analyzed, we propose the following optimum solutions, with the interval notation and meaning defined before:

- For fixed $\epsilon=0.080 \rightarrow$ relevant interval $m_{g}(\mathrm{MeV}):[300,600]$;

- For fixed $m_{g}=400 \mathrm{MeV} \rightarrow$ relevant interval $\epsilon:[0.080,0.090]$.

We note that the asymmetrical character of this solution is a consequence of the statistical analysis we have considered. Moreover, as we show in what follows, this solution allows us to estimate the uncertainties in the evaluated quantities in a consistent way and that is the point we are interested in here.

Table 2: Values of the fit parameters for $\epsilon=0.080 . C_{o}, C_{q q}, C_{q g}, C_{q g}^{\prime}$ and $C_{g g}$ are dimensionless and $\mu_{o}, \mu_{q q}, \mu_{q g}, \mu_{g g}$ are in $\mathrm{GeV}$.

\begin{tabular}{ccccc}
\hline$m_{g}(\mathrm{MeV}):$ & 300 & 400 & 500 & 600 \\
\hline$C_{o}$ & $1.170 \pm 0.026$ & $3.03 \pm 0.40$ & $5.61 \pm 0.24$ & $10.12 \pm 0.41$ \\
$C_{q q}$ & $3.8498 \pm 0.0036$ & $10.7 \pm 1.4$ & $30.65 \pm 0.72$ & $21.2 \pm 1.9$ \\
$C_{q g}\left(\times 10^{-1}\right)$ & $1.8993 \pm 0.0082$ & $8.74 \pm 0.59$ & $13.414 \pm 0.034$ & $42.70 \pm 0.62$ \\
$C_{q g}^{\prime}\left(\times 10^{-2}\right)$ & $1.8174 \pm 0.0068$ & $4.51 \pm 0.62$ & $18.842 \pm 0.060$ & $18.55 \pm 0.74$ \\
$C_{g g}\left(\times 10^{-3}\right)$ & $0.979 \pm 0.019$ & $3.79 \pm 0.17$ & $6.832 \pm 0.057$ & $18.20 \pm 0.26$ \\
$\mu_{o}$ & $0.1862 \pm 0.0041$ & $0.41 \pm 0.17$ & $0.196 \pm 0.021$ & $0.622 \pm 0.053$ \\
$\mu_{g g}$ & $0.6463 \pm 0.0055$ & $0.651 \pm 0.066$ & $0.6314 \pm 0.0031$ & $0.6427 \pm 0.0020$ \\
$\mu_{q q}$ & $0.156218 \pm 0.0023$ & $1.32 \pm 0.16$ & $1.574 \pm 0.047$ & $1.03 \pm 0.15$ \\
$\mu_{q g}$ & $0.8368 \pm 0.0027$ & $0.838 \pm 0.044$ & $0.8214 \pm 0.0020$ & $0.8377 \pm 0.0018$ \\
\hline
\end{tabular}

Let us first consider the case $\epsilon=0.080$ and $m_{g}(\mathrm{MeV})$ : [300,600]. The values of the fit parameters for $m_{g}=300,400,500$ and $600 \mathrm{MeV}$ are shown in Table II. With the extrema values $m_{g}=300 \mathrm{MeV}$ and $m_{g}$ $=600 \mathrm{MeV}$ for each evaluated quantity we plot the upper and the lower curves delimiting the region of uncertainty represented by a band, in the figures that follows. The results for $\sigma_{t o t}(s), \rho(s)$ are shown in Fig. 2 and those for the $\bar{p} p$ differential cross sections at $546 \mathrm{GeV}$ and $1.8 \mathrm{TeV}$ in Fig. 3. 


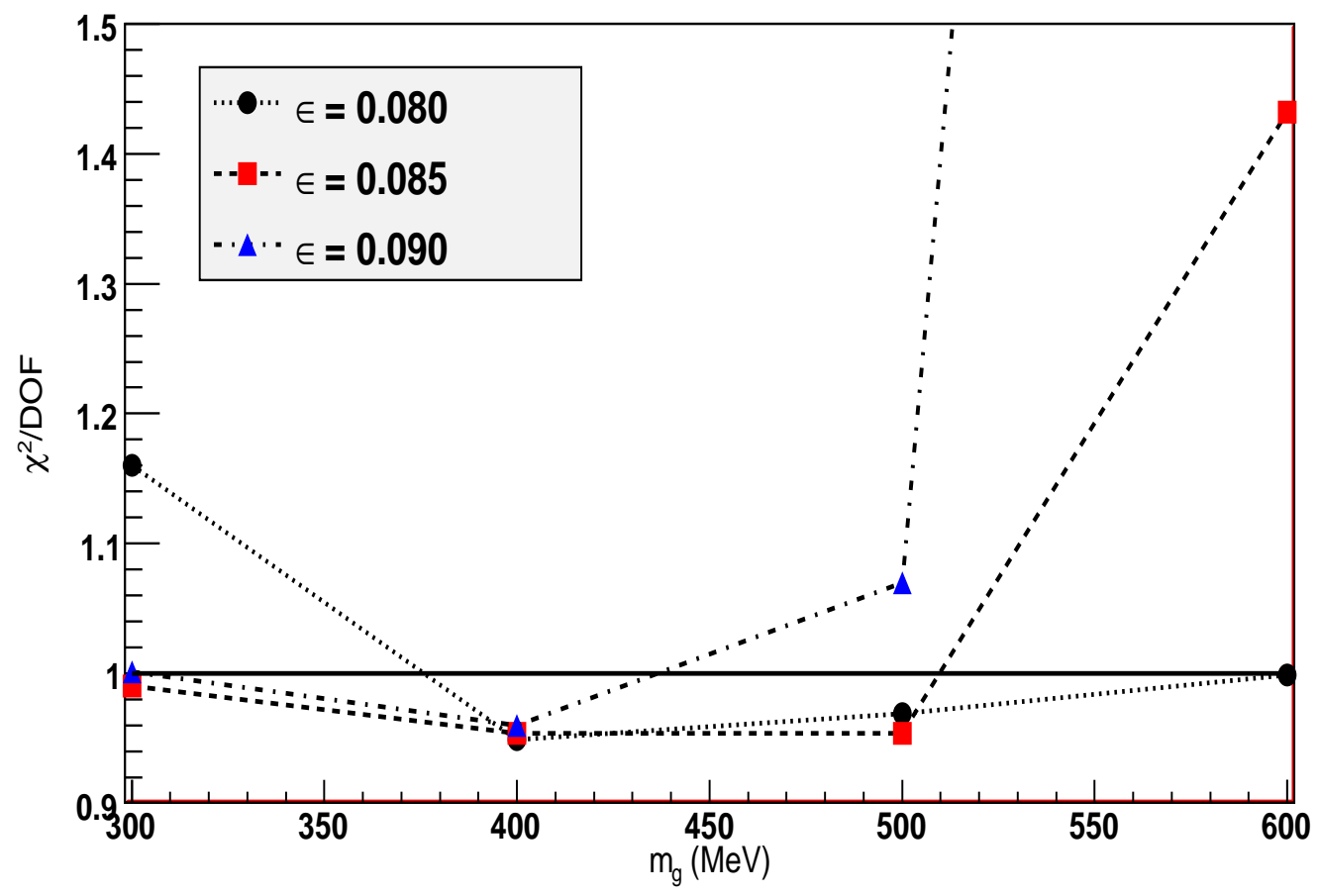

Figure 1: Reduced chi-square in terms of the dynamical gluon mass scale for different values of the soft Pomeron intercept. The straight line segments are drawn only to guide the eyes.

For further discussion, we have included in these figures some data that did not take part in the data reductions. In the plot of $\sigma_{\text {tot }}$ (Fig. 2.a) we have included the recent result by the TOTEM Collaboration at $7 \mathrm{TeV}$ [2] and in the differential cross section at $1.80 \mathrm{TeV}$ (Fig. 3.b) the data at $1.96 \mathrm{TeV}$ [46].

The predictions for the $p p$ differential cross section data at $7 \mathrm{TeV}$ and $14 \mathrm{TeV}$ are displayed in Fig. 4. In the former case $(7 \mathrm{TeV})$ the straight line segments and the point indicate recent experimental results obtained by the TOTEM Collaboration [1, 2] (see Table V): the optical point with the slope at the diffraction peak (dotted segment), the dip position (dashed vertical segment) and the value of the differential cross section at $q^{2}=0.7 \mathrm{GeV}^{2}$ (black square).

For the case $m_{g}=400 \mathrm{MeV}$ and $\epsilon:[0.080,0.090]$, the values of the fit parameters for $\epsilon=0.080,0.085$ and 0.090 are displayed in Table III. Here, with the extrema values $\epsilon=0.080$ and $\epsilon=0.090$ we plot the upper and the lower curves delimiting the region of uncertainty represented by a band in the figures that follows. The corresponding results for $\sigma_{t o t}(s), \rho(s)$ and the differential cross sections are shown in Figs. 5, 6 and 7.

\subsection{Discussion}

The main point in this section has been to propose estimations of the uncertainties in the evaluated quantities associated with relevant intervals for the fundamental parameters $\epsilon$ and $m_{g}$. As commented before we did not address the uncertainties associated with both intervals simultaneously but only an individual interval for a fixed parameter. Although that may represent an underestimation of the effective uncertainty it certainly gives more information on the reliability of the results than what could be obtained by fixing both parameters (or 6 as in 28]). In addition, in practice one band is practically contained in the other, so that the larger bands represent a reasonable result for our purposes.

The results presented in Tables I - III, figures 2, 3, 4 and 5, 6, 7 lead to some qualitative conclusions: 

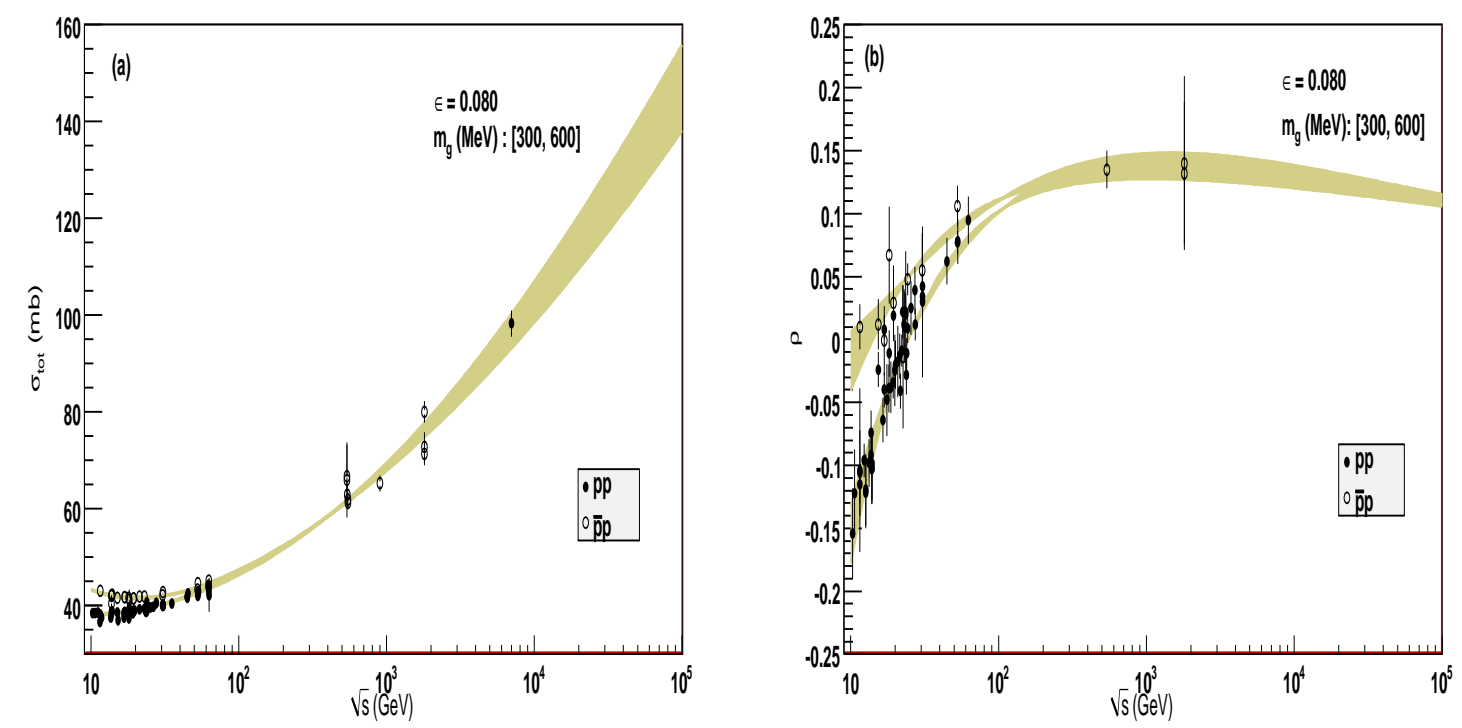

Figure 2: Total cross sections and $\rho$ parameter for fixed $\epsilon=0.080$, upper bound for $m_{g}=600 \mathrm{MeV}$ and lower bound for $m_{g}$ $=300 \mathrm{MeV}$.
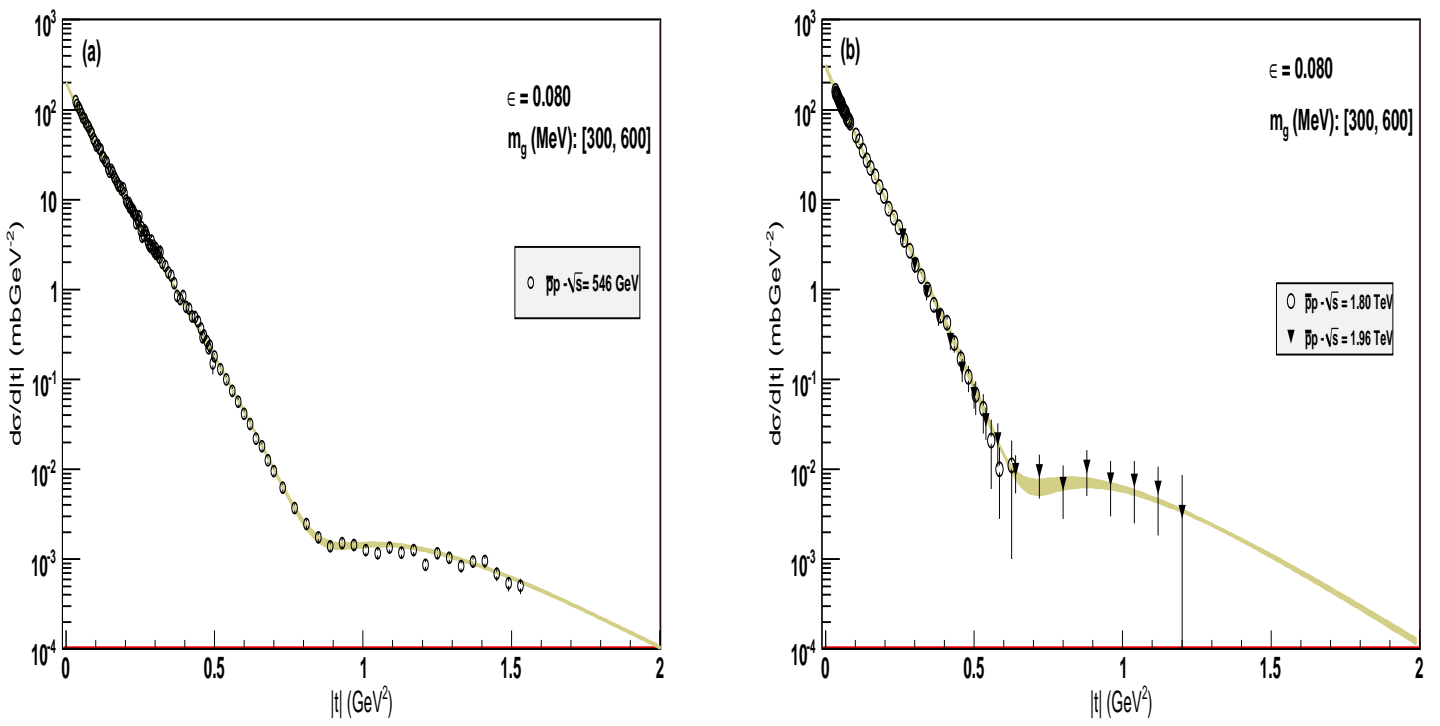

Figure 3: Differential cross sections at (a) $546 \mathrm{GeV}$ and (b) $1.8 \mathrm{TeV}$ (together with the $1.96 \mathrm{TeV}$ data) for fixed $\epsilon=0.080$, upper bound for $m_{g}=600 \mathrm{MeV}$ and lower bound for $m_{g}=300 \mathrm{MeV}$. 

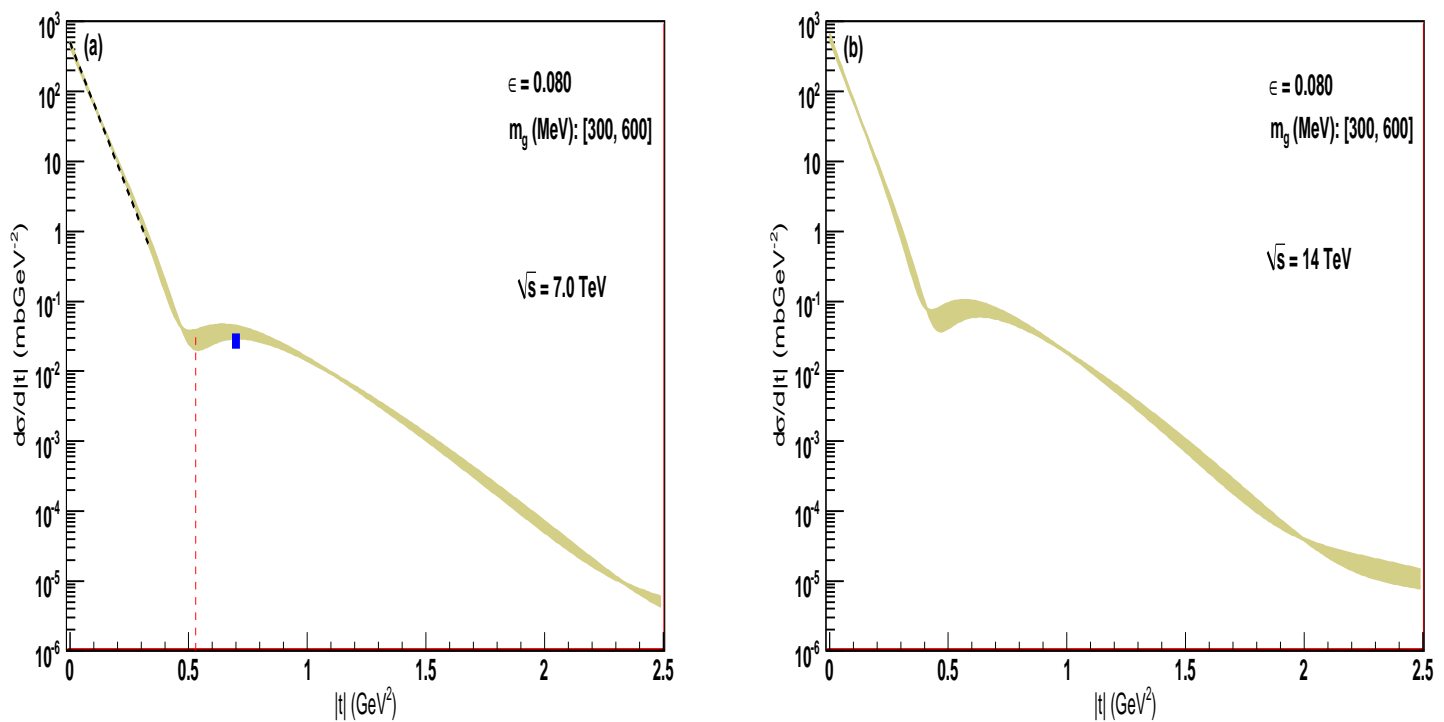

Figure 4: Predictions for the differential cross section at $7 \mathrm{TeV}$ (a) and $14 \mathrm{TeV}$ (b) for fixed $\epsilon=0.080$, upper bound for $m_{g}$ $=600 \mathrm{MeV}$ and lower bound for $m_{g}=300 \mathrm{MeV}$. At $7 \mathrm{TeV}$ the dotted segment indicate the optical point and slope at the diffraction peak, the dashed vertical segment the dip position and the black square the differential cross section at $q^{2}=0.7$ $\mathrm{GeV}^{2}$, as measured by TOTEM Collaboration (see Table V).
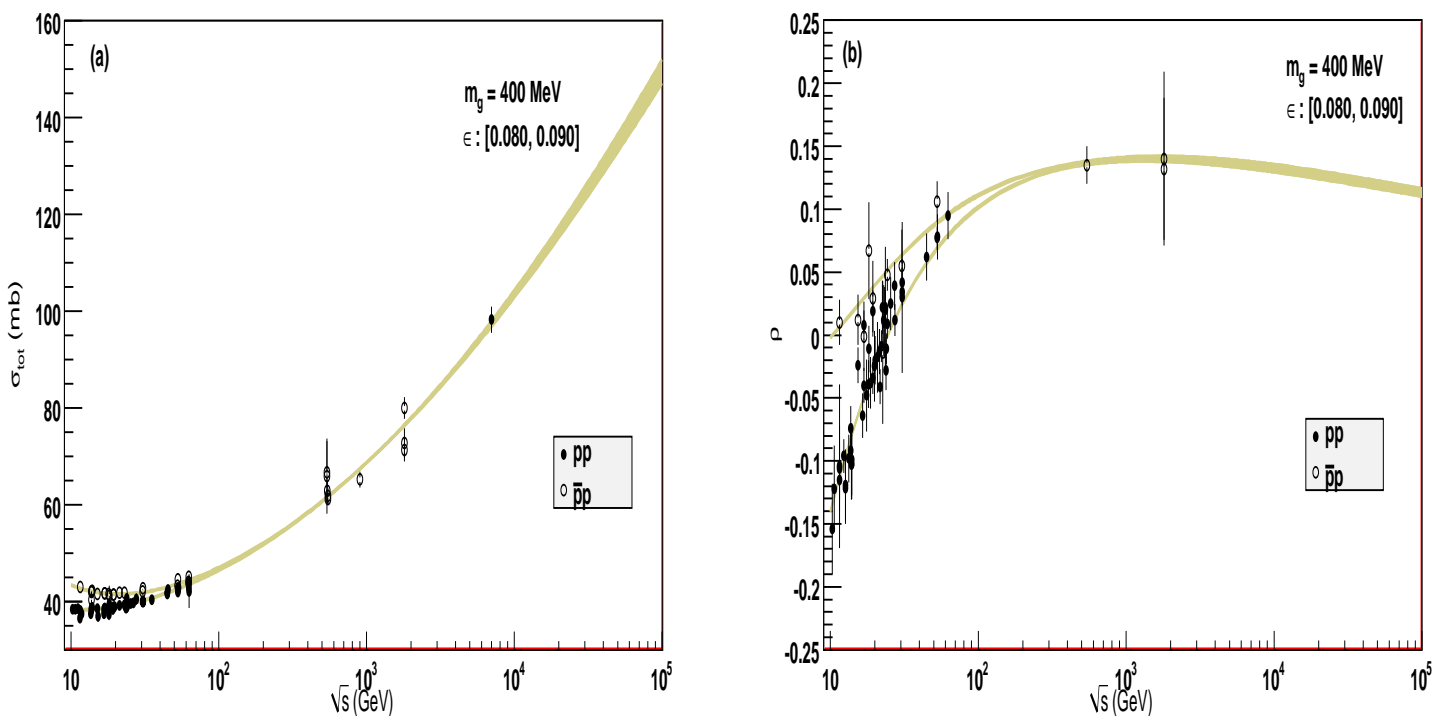

Figure 5: Total cross section and $\rho$ parameter for fixed $m_{g}=400 \mathrm{MeV}$, upper bound for $\epsilon=0.090$ and lower bound for $\epsilon=$ 0.080 . 

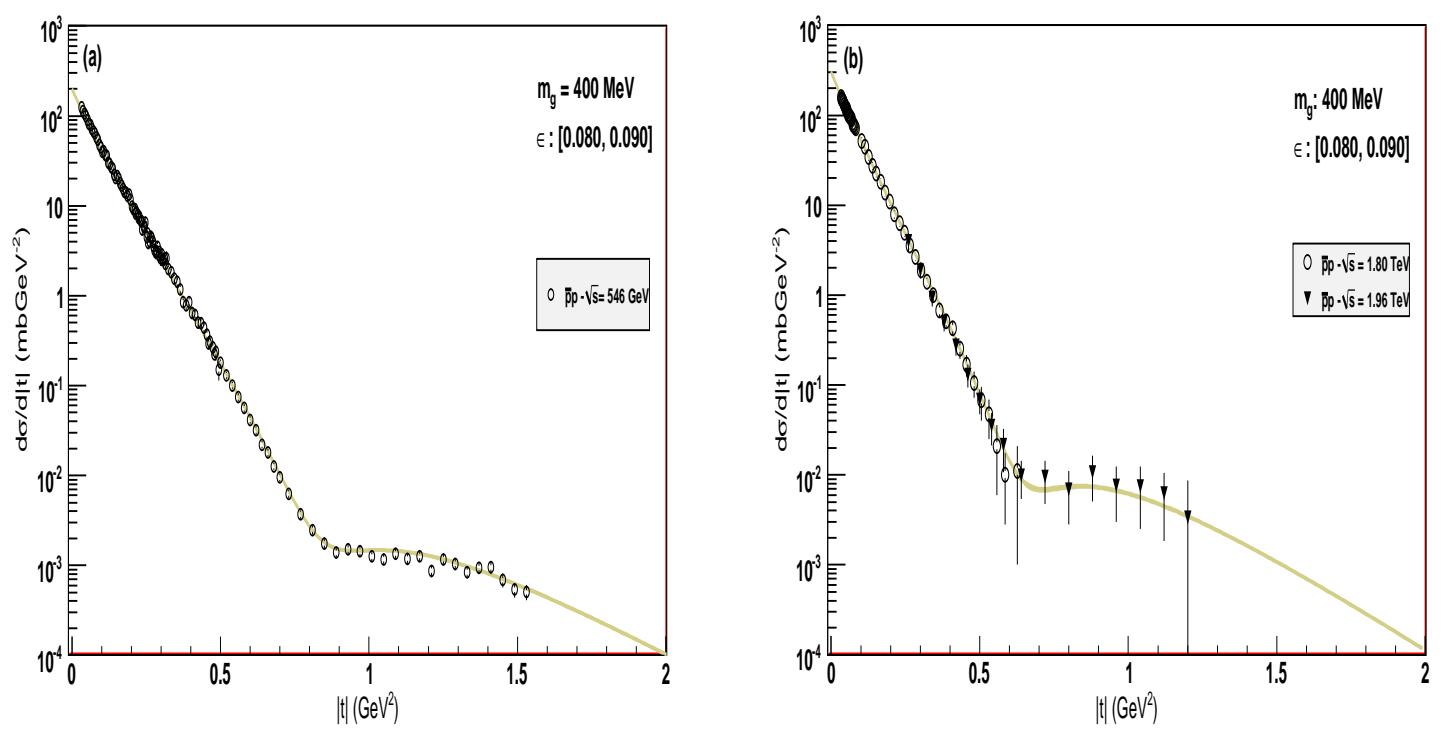

Figure 6: Differential cross sections at (a) $546 \mathrm{GeV}$ and (b) $1.8 \mathrm{TeV}$ (together with the data at $1.96 \mathrm{TeV}$ ) for fixed $m_{g}=400$ $\mathrm{MeV}$, upper bound for $\epsilon=0.090$ and lower bound for $\epsilon=0.080$.
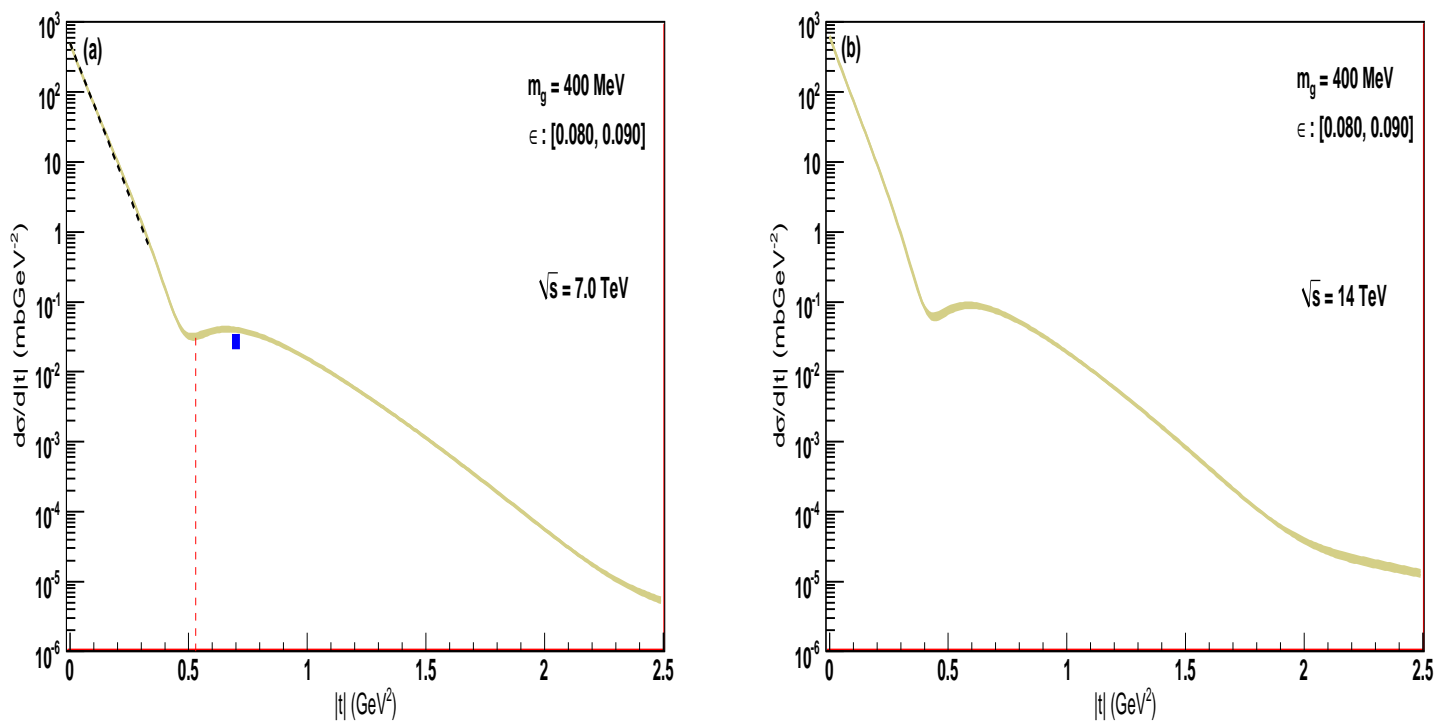

Figure 7: Predictions for the differential cross section at $7 \mathrm{TeV}$ (a) and $14 \mathrm{TeV}$ (b) for fixed $m_{g}=400 \mathrm{MeV}$, upper bound for $\epsilon=0.090$ and lower bound for $\epsilon=0.080$. Same legend as in Fig. 4. 
Table 3: Values of the fit parameters for $m_{g}=400 \mathrm{MeV} . C_{o}, C_{q q}, C_{q g}, C_{q g}^{\prime}$ and $C_{g g}$ are dimensionless and $\mu_{o}, \mu_{q q}, \mu_{q g}, \mu_{g g}$ are in $\mathrm{GeV}$.

\begin{tabular}{cccc}
\hline$\epsilon:$ & 0.080 & 0.085 & 0.090 \\
\hline$C_{o}$ & $3.03 \pm 0.40$ & $3.10 \pm 0.48$ & $3.11 \pm 0.42$ \\
$C q q$ & $10.7 \pm 1.4$ & $10.5 \pm 1.2$ & $10.2 \pm 1.1$ \\
$C_{q g}\left(\times 10^{-1}\right)$ & $8.74 \pm 0.59$ & $8.63 \pm 0.47$ & $8.66 \pm 0.41$ \\
$C_{q g}^{\prime}\left(\times 10^{-2}\right)$ & $4.51 \pm 0.62$ & $4.68 \pm 0.50$ & $4.69 \pm 0.45$ \\
$C_{g g}\left(\times 10^{-3}\right)$ & $3.79 \pm 0.17$ & $3.62 \pm 0.14$ & $3.49 \pm 0.12$ \\
$\mu_{o}$ & $0.41 \pm 0.17$ & $0.44 \pm 0.18$ & $4.45 \pm 0.16$ \\
$\mu_{g g}$ & $0.651 \pm 0.066$ & $0.6500 \pm 0.0065$ & $0.6496 \pm 0.0063$ \\
$\mu_{q q}$ & $1.32 \pm 0.16$ & $1.30 \pm 0.14$ & $1.27 \pm 0.16$ \\
$\mu_{q g}$ & $0.838 \pm 0.044$ & $0.8367 \pm 0.0040$ & $0.8367 \pm 0.0037$ \\
\hline
\end{tabular}

i. In addition to the good quality of the statistical results in terms of $\chi^{2} /$ DOF (Table I), Figs. 2,3 and 4 show that the visual description of all reduced data is quite good. In this energy region (up to 1.8 $\mathrm{TeV}$ ) the inferred error bands are not significant, which means that for any fixed values of $\epsilon$ and $m_{g}$ considered in the analysis, all data are quite well described. The correct reproduction of the highenergy differential cross section data up to $q^{2} \sim 1.5 \mathrm{GeV}^{2}$ (Figs. 3 and 6 ) seems to be a remarkable result in the context of QCD-inspired models.

ii. At higher energies (above those considered in the data reductions) the effects of the relevant intervals are significant since the general trend is an increase in the estimated error band above 1.8 TeV (Figs. 2 and 5).

iii. The error bands for fixed $m_{g}(400 \mathrm{MeV})$ are narrower than those for fixed $\epsilon(0.080)$ and the former are, in general, contained in the latter. That is a consequence of the difference in the relative intervals involved, $\sim 6 \%$ for $\epsilon$ and $\sim 30 \%$ for $m_{g}$, as well as the role played by each parameter in the formalism and data reductions.

iv. Table II and III illustrate the correlations among $\epsilon, m_{g}$ and the fit parameters. We see that this correlation is stronger in the case of $\epsilon$ fixed than in the case of $m_{g}$ fixed, an effect also associated with the difference in the relative intervals. Moreover, note that in both cases the correlations are stronger among the parameters associated with the normalization constants (the $C^{\prime} s$ ) than among those coming from the form factors (the $\mu^{\prime} s$ ).

v. Taking into account the error bands, the total cross section result by the TOTEM Collaboration at 7 $\mathrm{TeV}$ [2] is quite well described in both cases (Fig. 2.a and Fig. 5.a) and a reasonable consistency can be observed in the differential cross section up to the dip position (Fig. 4.a and Fig. 7.a).

vi. For the differential cross section at $14 \mathrm{TeV}$ a change of curvature is predicted around $q^{2}=2 \mathrm{GeV}^{2}$ in both cases (Fig. 4.b and Fig. 7.b).

Let us now present and discuss some quantitative results and predictions with the 12 variants considered, as well as the inferred upper and lower bounds for the evaluated quantities. For each one of the 12 variants the results for $\sigma_{t o t}, \rho, \sigma_{i n}$ and $\sigma_{e l} / \sigma_{t o t}$ at 7,14 and $57 \mathrm{TeV}$ are displayed in Table IV. As commented before, with the extrema values of $\epsilon$ or $m_{g}$ in each relevant interval, we evaluate the upper and lower bounds for all evaluated quantities. Table IV shows that in fact, for the values of $\epsilon$ and $m_{g}$ inside the relevant intervals the numerical values of the evaluated quantities lies inside the upper and lower bounds. Therefore, in what follows we shall give our predictions in terms of the band corresponding to these upper and lower bounds in each case ( $\epsilon$ or $m_{g}$ fixed). Since these bands have the same meaning as the relevant intervals (equally 
Table 4: Predictions for $p p$ integrated cross sections and $\rho$ parameter at the LHC and Auger energies for each tested value of $m_{g}$ and $\epsilon$. For each physical quantity the extrema values define the uncertainty band. The cross sections are in mb.

\begin{tabular}{|c|c|c|c|c|c|c|c|c|c|}
\hline $\begin{array}{l}\text { physical } \\
\text { quantity }\end{array}$ & $\begin{array}{c}\epsilon=0.080 \\
m_{g}(\mathrm{MeV}):\end{array}$ & 300 & 400 & 500 & 600 & $\begin{array}{c}m_{g}=400 \mathrm{MeV} \\
\epsilon:\end{array}$ & 0.080 & 0.085 & 0.090 \\
\hline$\sigma_{i n}(7 \mathrm{TeV})$ & & 70.0 & 72.3 & 70.6 & 74.6 & & 72.3 & 72.6 & 73.0 \\
\hline$\sigma_{i n}(14 \mathrm{TeV})$ & & 76.7 & 79.8 & 77.5 & 82.9 & & 79.8 & 80.3 & 81.0 \\
\hline$\sigma_{i n}(57 \mathrm{TeV})$ & & 91.4 & 96.1 & 92.8 & 100.9 & & 96.1 & 97.2 & 98.4 \\
\hline$\sigma_{\text {tot }}(7 \mathrm{TeV})$ & & 93.2 & 96.9 & 94.0 & 100.2 & & 96.9 & 97.4 & 98.0 \\
\hline$\sigma_{\text {tot }}(14 \mathrm{TeV})$ & & 103.9 & 108.8 & 104.9 & 113.4 & & 108.8 & 109.6 & 110.6 \\
\hline$\sigma_{t o t}(57 \mathrm{TeV})$ & & 127.8 & 135.6 & 129.5 & 143.1 & & 135.6 & 137.3 & 139.4 \\
\hline$\sigma_{e l} / \sigma_{t o t}(7 \mathrm{TeV})$ & & 0.2489 & 0.2539 & 0.2489 & 0.2554 & & 0.2539 & 0.2546 & 0.2551 \\
\hline$\sigma_{e l} / \sigma_{t o t}(14 \mathrm{TeV})$ & & 0.2618 & 0.2665 & 0.2612 & 0.2690 & & 0.2665 & 0.2673 & 0.2676 \\
\hline$\sigma_{e l} / \sigma_{t o t}(57 \mathrm{TeV}$ & & 0.2848 & 0.2834 & 0.2834 & 0.2949 & & 0.2834 & 0.2921 & 0.2941 \\
\hline$\rho(7 \mathrm{TeV})$ & & 0.1225 & 0.1321 & 0.1245 & 0.1409 & & 0.1321 & 0.1346 & 0.1376 \\
\hline$\rho(14 T e V)$ & & 0.1189 & 0.1272 & 0.1208 & 0.1349 & & 0.1272 & 0.1299 & 0.1330 \\
\hline
\end{tabular}

likely values), we shall use the same notation, namely the bounds inside brackets and separated by a comma: [lower-bound, upper-bound].

Let us consider first the $p p$ elastic scattering at $7 \mathrm{TeV}$ with focus on the published results by the TOTEM Collaboration [1, 2]. Our predictions (bands) for several physical quantities are displayed in Table V, together with the corresponding results by the TOTEM Collaboration. From that Table and within the error bands we are led to the conclusions that follows.

- For $\epsilon=0.080$ the predictions are: (a) consistent with all cross sections, optical point and dip position; (b) barely consistent with the forward slope; (c) not consistent with the slope near the dip position, the differential cross section at $q^{2}=0.7 \mathrm{GeV}^{2}$ and the exponent $n$ in the power law at large momentum transfer.

- For $m_{g}=400 \mathrm{MeV}$ the predictions are: (a) consistent with all the cross sections and the optical point; (b) barely consistent with the dip position; (c) not consistent with both slopes, the differential cross section at $q^{2}=0.7 \mathrm{GeV}^{2}$ and the exponent $n$.

How good or bad are these results? In [1] the TOTEM Collaboration displays in Table 4 the predictions from some representative phenomenological models compiled in [47]. Comparison of our results with these predictions shows that despite all the above inconsistencies, our efficiency in the description of the experimental data is compatible with that presented by all quoted models. It is also interesting to note in that Table that our wrong prediction for the exponent in the power law at large $q^{2}$, namely $n \sim 10.5$, is in plenty agreement with the prediction by Block et al: $n=10.4$. This agreement may be related with the choice of four dipole form factors, present in both models. Moreover, the first TOTEM data on differential elastic cross section at $7 \mathrm{TeV}$ has been measured in specialized runs of LHC and in the momentum transfer range of $0.36<-t<2.5 \mathrm{GeV}^{2}$. Notice that none of the representative models for elastic scattering can fully reproduce its overall features; in special, the dip position, dip depth and large-t behaviour [1]. Particularly in the range $1.5<-t<2.0 \mathrm{GeV}^{2}$ the data presented a power law pattern proportional to $\sim|t|^{-8}$, which is in poorly agreement with general model predictions. Interestingly enough, this specific power law behaviour was first envisaged by Donnachie and Landshoff [48] in a model which accounts for the contribution of three gluon exchange in elastic scattering at high energies. Yet, in the QCD context, the constituent interchange model by Lepage and Brodsky [49] predicts a different power law behaviour, namely $\sim|t|^{-10}$. The DGM model results at the large- $t$ region are consistent with the latter. We shall return to this point in our conclusions.

At other energies (14 and $57 \mathrm{TeV}$ ) the bands in our predictions also correspond to the extrema values for each physical quantity, as given in Table IV, for the cases $\epsilon=0.080$ and $m_{g}=400 \mathrm{MeV}$. For example, 
at $57 \mathrm{TeV}$ we predict $\sigma_{\text {tot }}(\mathrm{mb}):[127.8,143.1]$ for $\epsilon=0.080$ and $\sigma_{\text {tot }}(\mathrm{mb}):[127.8,139.4]$ for $m_{g}=400$ $\mathrm{MeV}$. We note that these results are consistent with recent estimation of this quantity through an analytical parametrization, $\sigma_{t o t}=133.4 \pm 1.6 \mathrm{mb}[50]$.

Table 5: TOTEM results at $7 \mathrm{TeV}$ with systematic and statistical error added in quadrature and the uncertainty band predictions.

\begin{tabular}{|c|c|c|c|}
\hline \hline $\begin{array}{c}\text { Physical } \\
\text { quantity }\end{array}$ & $\begin{array}{c}\text { TOTEM } \\
\text { results }\end{array}$ & $\begin{array}{c}\epsilon=0.080 \\
m_{g}(\mathrm{MeV}):[300,600]\end{array}$ & $\begin{array}{l}m_{g}=400 \mathrm{MeV} \\
\epsilon:[0.080,0.090]\end{array}$ \\
\hline $\mathrm{B}\left(0.36 \leq t \leq 0.47 \mathrm{GeV}^{2}\right)\left(\mathrm{GeV}^{-2}\right)[1]$ & $23.60 \pm 0.64$ & {$[19.8,22.4]$} & {$[20.8,21.6]$} \\
\hline$\left|t_{d i p}\right|\left(\mathrm{GeV}^{2}\right)[1]$ & $0.53 \pm 0.01$ & {$[0.51,0.54]$} & {$[0.51,0.52]$} \\
\hline$n$ in $|t|^{-n}\left(1.5 \leq t \leq 2.0 \mathrm{GeV}^{2}\right)[1]$ & $7.80 \pm 0.32$ & {$[10.1,10.6]$} & {$[10.5,10.5]$} \\
\hline$\frac{d \sigma}{d|t|}(|t|=0.7)\left(\mathrm{mbGeV}{ }^{-2}\right)[1]$ & $2.70_{-0.58}^{+0.71} \times 10^{-2}$ & {$[3.0,4.3] \times 10^{-2}$} & {$[3.8,4.1] \times 10^{-2}$} \\
\hline$\sigma_{e l}(\mathrm{mb})[2]$ & $24.8 \pm 1.2$ & {$[23.2,25.6]$} & {$[24.6,25.0]$} \\
\hline$\sigma_{\text {in }}(\mathrm{mb})[2]$ & $73.5_{-1.4}^{+1.9}$ & {$[70.0,74.6]$} & {$[72.3,73.0]$} \\
\hline$\sigma_{t o t}(\mathrm{mb})[2]$ & $98.3 \pm 2.8$ & {$[93.2,100.2]$} & {$[96.9,98.0]$} \\
\hline $\mathrm{B}\left(0.02 \leq t \leq 0.33 \mathrm{GeV}^{2}\right)\left(\mathrm{GeV}^{-2}\right)[2]$ & $20.10 \pm 0.36$ & {$[18.6,19.8]$} & {$[19.3,19.4]$} \\
\hline$\left.\frac{d \sigma}{d \mid t}\right|_{t=0}\left(\mathrm{mbGeV}^{-2}\right)[2]$ & $504 \pm 27$ & {$[451,523]$} & {$[488,500]$} \\
\hline \hline
\end{tabular}

At last, let us discuss our two proposed independent solutions for the inference of uncertainties. Guided by the TOTEM results at $7 \mathrm{TeV}$, Table $\mathrm{V}$ suggests that the case of fixed $\epsilon=0.080$ presents better consistence with the experimental data than the case of fixed $m_{g}=400 \mathrm{MeV}$. Since we shall not treat the simultaneous effects of both parameters, we may conclude that by fixing $\epsilon$ at the above value we arrive at a best solution. Indeed this conclusion is corroborated by the following facts:

- The bands for fixed $m_{g}$ lies, in general, inside those for fixed $\epsilon$ and therefore the former may be seen as a particular case of the latter.

- The fixed value $\epsilon=0.080$ for the soft Pomeron intercept is consistent with all phenomenological analyses of the elastic hadron scattering, in particular with those treating constraint and extrema bounds [44, 45].

- The interval $m_{g}(\mathrm{MeV}):[300,600]$ is consistent with all phenomenological and theoretical results on the dynamical gluon mass.

Therefore, we understand that our approach, as it has been presented here, has an optimum solution with only one fixed parameter, $\epsilon=0.080$ and a relevant physical interval for the dynamical gluon mass scale, $m_{g}$ $(\mathrm{MeV}):[300,600]$. In this case, within the inferred error bands the predictions present reasonable consistency with the TOTEM results at $7 \mathrm{TeV}$, at least at the same level of some representative phenomenological models.

\section{Conclusions and Final remarks}

Further developments on a dynamical gluon mass approach to elastic $p p$ and $\bar{p} p$ scattering have been presented and discussed. The main conceptual ingredient, introduced in [8, 9], concerns the dynamical gluon mass scale as a natural regulator for the IR divergences associated with the gluon-gluon cross section, leading to the identification of explicit nonperturbative contributions to high-energy elastic hadron scattering. In this context a consistent physical meaning is given to two ad hoc parameters, typical of mini-jet or QCDinspired models: the infrared mass scale and the effective value of the running coupling constant.

In this paper the main novel results concern the account of the energy dependencies in the dynamical gluon mass, Eq. (8), the detailed investigation on the influence in the predictions from relevant physical intervals for the dynamical gluon mass scale and the soft Pomeron intercept, as well as the introduction of a method to estimate the corresponding bands of uncertainty in all evaluated quantities. Fits to an improved data ensemble on $p p$ and $\bar{p} p$ elastic scattering up to $1.8 \mathrm{TeV}$ have led to quite good descriptions of all reduced data, including the high-energy differential cross section up to $q^{2} \sim 1.5 \mathrm{GeV}^{2}$. With the inferred uncertainty bands the experimental data recently obtained by the TOTEM Collaboration are reasonably 
well described, except the region beyond the dip position. Comparison with the predictions from some representative phenomenological models, presented and quoted in [1], shows that our results are at the same level of consistency with the TOTEM data, with the advantage in our case of explicit connections with nonperturbative QCD. As commented in Appendix A, our data reductions disfavor a soft solution for the dynamical gluon mass.

The uncertainty in the evaluated quantities, associated with relevant intervals for the fundamental parameters $\epsilon$ and $m_{g}$, seems to us a fundamental information for reliable phenomenological predictions. Although we did not address the question associated with both intervals simultaneously, we understand that our proposed estimation of the bounds gives more information on the reliability of the results than what could be obtained by fixing both parameters. Based on the results and discussion displayed in Subsection 3.3, we propose as our best present solution the case of fixed $\epsilon=0.080$ and interval $m_{g}(\mathrm{MeV})$ : [300, 600]. With that we have only one fixed parameter, with well founded physical justification.

The critical point raised in respect to fixing parameters have been directed to a class of QCD inspired models. However the criticism certainly apply to any phenomenological model with fixed parameters whose numerical values do not have an explicit physical justification and its consequences in the evaluated quantities are not investigated or even discussed.

In the phenomenological context, model developments are in general more important than any particular formulation and, in our case, at least two fundamental aspects demand further investigation. The first one concerns the phenomenological choice for the gluon distribution function, Eq. (18), which introduces the Pomeron intercept as a fundamental parameter. Despite its efficiency in the analysis we have developed, it may be important to study the effects of different parton density functions, along the lines that have been discussed by Achilli et al. [43]. In this class of QCD-based models, the attenuation in the rise of the total cross sections comes from soft gluon emission from colliding partons [5, 6, 7]. These emissions affect the matter distribution and the resulting overlap function can be calculated from the soft gluon transverse-momentum resummed distribution. In this $k_{T}$ resummation scheme the overlap function in b-space depends upon the behaviour of the coupling $\alpha_{s}\left(k_{T}\right)$ as $k_{T} \rightarrow 0$, being the calculation only possible after the introduction of a phenomenological infrared modification for $\alpha_{s}\left(k_{T}\right)$. In the context of the DGM approach, it should be stressed that the QCD effective charge (7) is obtained from the QCD Lagrangian, i.e., it is derived from first principles, in a scenario where infrared effects are naturally taken into account. Hence this effective strong coupling could be naturally embodied in a soft $k_{T}$ resummation scheme in order to avoid infrared divergences. A second aspect is related to the choice of form factors as simple dipole parametrizations. As commented before, the fact that our prediction for the exponent in the power law at large momentum transfer is near 10 (as is the case with the Aspen model), suggest that this result is directly related with the above choice for the form factors. Since deviations from the dipole parametrization have been indicated in model-independent analyses of $p p$ elastic scattering [51, 52, 53], to take into account these empirical results may give new insights in the predictions at large momentum transfer. We are presently investigating the above two lines.

\section{Appendix A}

With the DGM approach described in Sect. 2, in addition to the Cornwall's solution (8), we have also considered the possibility of a soft behavior for the dynamical gluon mass. A power-law running behavior for $M_{g}^{2}(\hat{s})$ was first envisaged in [54] and according to an OPE calculation the most probable asymptotic behavior of the running gluon mass is proportional to $1 / \hat{s}$ [55]. At the level of a non-linear Schwinger-Dyson equation this asymptotic behavior is given by

$$
M_{g}^{2}(\hat{s})=\frac{m_{g}^{4}}{\hat{s}+m_{g}^{2}}\left[\frac{\ln \left(\frac{\hat{s}+m_{g}^{2}}{\Lambda^{2}}\right)}{\ln \left(\frac{\tau m_{g}^{2}}{\Lambda^{2}}\right)}\right]^{\gamma_{2}-1}
$$


where

$$
\gamma_{2}=\frac{4}{5}+\frac{6 c_{1}}{5}
$$

$c_{1} \in[0.7,1.3]$ and the parameters $\tau$ and $m_{g}$ are constrained to lie in the interval $[1.0,8.0]$ and [300, 600 $\mathrm{MeV}$ ], respectively [56]. However, with the procedure described in Subsect. 3.A, this solution leads to much worse $\chi^{2} /$ DOF compared to the ones related to Eq. (8), which disfavor such solution.

\section{Acknowledgments}

Research supported by CNPq (A.A.N) and FAPESP (D.A.F and M.J.M).

\section{References}

[1] G. Antchev et al. (TOTEM Collaboration) Europhys. Lett. 9541001 (2011)

[2] G. Antchev et al. (TOTEM Collaboration), Europhys. Lett. 9621002 (2011)

[3] D. Cline, F. Halzen, J. Luthe, Phys. Rev. Lett. 54, 757 (1985); T.K. Gaisser and F. Halzen, Phys. Rev. Lett. 54, 1754 (1985); P. L'Heureux, B. Margolis, P. Valin, Phys. Rev. D 32, 1681 (1985); G. Pancheri and Y.N. Srivastava, Phys. Lett. B 159, 69 (1985); G. Pancheri and Y.N. Srivastava, Phys. Lett. B 182, 199 (1986); L. Durand and H. Pi, Phys. Rev. Lett. 58, 303 (1987); A. Capella, J. Tran Thanh Van, J. Kwiecinski, Phys. Rev. Lett. 58, 2015 (1987); J. Dias de Deus, J. Kwiecinski, Phys. Lett. B 196, 537 (1987); L. Durand and H. Pi, Phys. Rev. D 38, 78 (1988).

[4] F. Nemes, T. Csorgo, arXiv:1202.2438 [hep-ph]; F. Nemes, T. Csorgo, arXiv:1204.5617 [hep-ph]; A.D. Martin, M.G. Ryskin, V. A. Khoze, arXiv:1110.1973 [hep-ph].

[5] A. Corsetti, A. Grau, G. Pancheri, and Y. N. Srivastava, Phys. Lett. B 382, 282 (1996).

[6] A. Grau, G. Pancheri, and Y. Srivastava, Phys.Rev. D 60, 114020 (1999).

[7] R. M. Godbole, A. Grau, G. Pancheri, and Y. N. Srivastava, Phys. Rev. D 72, 076001 (2005).

[8] E.G.S. Luna, A.F. Martini, M.J. Menon, A. Mihara, and A.A. Natale, in: AIP Conference Proceedings, vol. 739, American Institute of Physics, New York, 2004, p. 572.

[9] E.G.S. Luna, A.F. Martini, M.J. Menon, A. Mihara, and A.A. Natale, Phys. Rev. D 72, 034019 (2005).

[10] E.G.S. Luna and Natale, Phys. Rev. D 73, 074019 (2006).

[11] F.D.R. Bonnet, et al., Phys. Rev. D 64 (2001) 034501; A. Cucchieri, T. Mendes, A. Taurines, Phys. Rev. D 67 (2003) 091502(R); P.O. Bowman, et al., Phys. Rev. D 70 (2004) 034509; A. Sternbeck, E.-M. Ilgenfritz, M. Muller-Preussker, A. Schiller, Phys. Rev. D 72 (2005) 014507; A. Sternbeck, E.-M. Ilgenfritz, M. Muller-Preussker, Phys. Rev. D 73 (2006) 014502; Ph. Boucaud, et al., JHEP 0606 (2006) 001; P.O. Bowman, et al., hep-lat/0703022 I.L. Bogolubsky, E.M. Ilgenfritz, M. Muller-Preussker, A. Sternbeck, Phys. Lett 676 (2009) 69; O. Oliveira, P. J. Silva, arXiv:0910.2897 [hep-lat]; O. Oliveira, P. J. Silva, arXiv:0911.1643 [hep-lat]; A. Cucchieri, T. Mendes, E.M.S. Santos, Phys. Rev. Lett. 103 (209) 141602; A. Cucchieri, T. Mendes, Phys. Rev. D 81 (2010) 016005; D. Dudal, O. Oliveira, N. Vandersickel, Phys. Rev. D 81 (2010) 074505.

[12] F. Halzen, G. Krein, A.A. Natale, Phys. Rev. D 47, 295 (1993); M.B. Gay Ducati, F. Halzen, A.A. Natale, Phys. Rev. D 48, 2324 (1993); A.C. Aguilar, A. Mihara, A.A. Natale, Phys. Rev. D 65, 054011 (2002); E.G.S. Luna, Phys. Lett. B 641, 171 (2006); E.G.S. Luna, Braz. J. Phys. 37 (2007) 84; E.G.S. Luna, in: AIP Conference Proceedings, vol. 1296, American Institute of Physics, New York, 2010, p. 183; E.G.S. Luna, A.L. dos Santos, in: AIP Conference Proceedings, vol. 1296, American Institute of Physics, New York, 2010, p. 330.

[13] E.G.S. Luna, A.A. Natale and C.M. Zanetti, Int. J. Mod. Phys. A 23, 151 (2008).

[14] E.G.S. Luna, A.A. Natale, and A.L. dos Santos, Phys. Lett. B 698, 52 (2011).

[15] D.A. Fagundes, E.G.S. Luna, M.J. Menon, A.A. Natale, Testing parameters in an eikonalized dynamical gluon mass model, arXiv:1108.1206 [hep-ph].

[16] V. Barone and E. Predazzi, High-Energy Particle Diffraction (Spring-Verlag, Berlin, 2002).

[17] J. M. Cornwall, Phys. Rev. D 26, 1453 (1982).

[18] J. M. Cornwall, J. Papavassiliou, Phys. Rev. D 40, 3474 (1989);

[19] J. Papavassiliou and J. M. Cornwall, Phys. Rev. D 44, 1285 (1991)

[20] D. Binosi and J. Papavassiliou, Phys. Rept. 479, 1 (2009).

[21] A.C. Aguilar, A. Mihara and A.A. Natale, Int. J. Mod. Phys. A 19, 249 (2004).

[22] A.C. Aguilar and A.A. Natale, J. High Energy Phys. 8, 57 (2004).

[23] L.V. Gribov, E.M. Levin, and M.G. Ryskin, Phys. Rep. 100, 1 (1983).

[24] E.M. Levin and M.G. Ryskin, Phys. Rep. 189, 267 (1990).

[25] B. Margolis, P. Valin, M.M. Block, F. Halzen and R.S. Fletcher, Phys. Lett. B 213, 221 (1988).

[26] M. Block, R. Fletcher, F. Halzen, B. Margolis and P. Valin, Nucl. Phys. B (Proc. Suppl.) 12, 238 (1990).

[27] M. M. Block, F. Halzen, B. Margolis, Phys. Rev. D 45, 839 (1992).

[28] M. M. Block, E. M. Gregores, F. Halzen, G. Pancheri, Phys. Rev. D 60, 054024 (1999).

[29] M. M. Block, Phys. Rep. 436 (2006) 71-215.

[30] T.T. Wu and C.N. Yang, Phys. Rev. B 137, 708 (1965).

[31] T.T. Chou and C.N. Yang, Phys. Rev. 170, 1591 (1968). 
[32] T.T. Chou and C.N. Yang, Phys. Rev. Lett. 20, 1213 (1968).

[33] L. Durand and R. Lipes, Phys. Rev. Lett. 20, 637 (1968).

[34] H.M. Georgi, S.L. Glashow, M.E. Machacek, and D.V. Nanopoulos, Annals of Phys. 114, 273 (1978).

[35] J.F. Owens, E. Reya, and M. Glück, Phys. Rev. D 18, 1501 (1978).

[36] R. Cutler and D. Sivers, Phys. Rev. D 17, 196 (1978).

[37] R.F. Ávila, M.J. Menon, Nucl Phys. A, 744, 249 (2004).

[38] K. Eggert, TOTEM Status Report and First Measurement of the Total Cross-section, 107th LHCC Meeting, http://totem.web.cern.ch/totem/

[39] S. Bültmann et al. (pp2pp Collaboration), Phys. Lett. B 579, 245 (2004).

40] K. Nakamura et al. (Particle Data Group), J. Phys. G 37, 075021 (2010).

[41] Durham Reaction Database/http://durpdg.dur.ac.uk/HEPDATA/REAC

[42] http://root.cern.ch/drupal/, http://root.cern.ch/root/html/TMinuit.html.

43] A. Achilli, Y. Srivastava, R. Godbole, A. Grau, G. Pancheri and O. Shekhovtsova, Total and inelastic cross-sections at LHC at $\sqrt{s}=7 \mathrm{TeV}$ and beyond, arXiv:1102.1949 [hep-ph] and references therein.

44] E. G. S. Luna, M. J. Menon, Phys. Lett. B 565, 123 (2003).

[45] E. G. S. Luna, M. J. Menon, J. Montanha, Nucl. Phys. A 745, 104 (2004).

[46] D0 Collaboration, A. Brandt, PoS DIS2010 059(2010).

[47] J. Kašpar V. Kundrát, M. Lokajíček and J. Procházka, Nucl. Phys. B 843, 84 (2011).

[48] A. Donnachie and P. V. Landshoff, Z. Phys. C 2, 55 (1979); A. Donnachie and P. V. Landshoff, Phys. Lett. B 387,637 (1996).

[49] G. P. Lepage and S. J. Brodsky, Phys. Rev. D 22, 2157 (1980).

[50] M. M. Block and F. Halzen, Phys. Rev. D 72, 036006 (2005).

[51] D.A. Fagundes and M.J. Menon, Int. J. Mod. Phys. A 26, 3219 (2011).

[52] R.F. Ávila and M.J. Menon, Eur. Phys. J. C 54, 555 (2008).

[53] P.A.S. Carvalho, A.F. Martini and M.J. Menon, Eur. Phys. J. C 39, 359 (2005).

54] J. M. Cornwall and W. S. Hou, Phys. Rev. D 34, 585 (1986).

[55] M. Lavelle, Phys. Rev. D 44, 26 (1991); D. Dudal, J.A. Gracey, S. P. Sorella, N. Vandersickel, H. Verschelde, Phys. Rev. D 78, 065047 (2008).

[56] A. C. Aguilar and J. Papavassiliou, Eur. Phys. J. A 35, 189 (2008). 